\title{
Quantum cosmology and the equation Schwinger - Tomonaga.
}

\author{
Kuyukov Vitaliy P. \\ vitalik.kayukov@mail.ru \\ SFU, Russia
}

\begin{abstract}
In this article we will use the recent approach to quantum cosmology based on the principle of uncertainty between the Hubble parameter and the volume of the space. In this article, we use the complete formulation of the equations for the wave function of the universe. For this purpose, the Schwinger equation for the evolution of the wave function along the hyper-surface of space-time.
\end{abstract}

In quantum mechanics, the limit of measurement accuracy is installed between the speed and the position of the particle.

$$
\Delta v \Delta x \geq \frac{h}{m}
$$

This ratio is applicable for small subsystems, but for cosmology, this ratio does not make much sense in the context of the entire Universe.

Therefore, we consider two quantities cosmology: the Hubble parameter $\mathrm{H}$ and the volume of space $\mathrm{V}$. The first value of the Hubble parameter, is a measure of the speed of expansion of space.

$$
H=\frac{1}{a} \frac{d a}{d t}
$$

Please note that, based on dimensional analysis, multiplication of the Hubble parameter on the volume of space gives constant, that is, a combination of the gravitational constant, the speed of light and Planck's constant.

$$
[H][V]=\left[\frac{G h}{c^{2}}\right]
$$

Hence, we can assume the ratio. The uncertainty principle between the Hubble parameter and the volume of the space.

$$
\Delta H \Delta V \geq \frac{4 \pi G h}{c^{2}}
$$

The more defined the space, the less accurate is the Hubble parameter (the rate of expansion of space) and vice versa.

This ratio is a property of the phase space $\mathrm{CVH}$ - algebra considered in earlier works on quantum cosmology $[10,12,14]$. 
The uncertainty principle gives the required quantum mechanical definition of a wave function.

$$
\Psi(V)=A e^{-i \frac{c^{2}}{4 \pi G h} \int H d V}
$$

This allows you to determine the energy density

$$
\frac{d E}{d V}=\frac{3 c^{2} H^{2}}{8 \pi G}
$$

The wave equation

$$
\frac{6 \pi G h^{2}}{c^{2}} \frac{\partial^{2} \Psi}{\partial V^{2}}+\frac{d E}{d V} \Psi=0
$$

This is quite interesting, we get the analog of the stationary Schrödinger equation is second order in volume. The wave function is a function of the volume of the space.

In this article, we use the complete formulation of the interim equations for the wave function of the universe. For this purpose, the equation Schwinger for the evolution of the wave function through the hyper-surface of space-time.

Consider the time equation of Schrodinger.

$$
i h \frac{\partial \Psi}{\partial t}=E \Psi
$$

The Schwinger equation summarizes the evolution of the wave function through the hyper-surface the space-time. Where is the Hamiltonian operator of the energy density.

$$
\begin{gathered}
i h \frac{\partial \Psi}{\partial \Omega}=\frac{d E}{d V} \Psi \\
d \Omega=d t d V
\end{gathered}
$$

This equation is incomplete.

Using the uncertainty relation, we find that the operator of the energy density can be determined through the derivatives of the volume space.

This allows us to derive a General equation for the evolution of the wave function through the Hypersurface of space-time. Where the wave function itself is a function of volume and time.

$$
i h \frac{\partial \Psi}{\partial \Omega}=-\frac{6 \pi G h^{2}}{c^{2}} \frac{\partial^{2} \Psi}{\partial V^{2}}
$$

General equation ( metric and volume)

$$
\begin{gathered}
\partial V_{i k}=\partial g_{i k} d V \\
g_{i k \alpha \beta}-\text { metric Wheeler }
\end{gathered}
$$




$$
i h \frac{\partial \Psi}{\partial \Omega}=-\frac{6 \pi G h^{2}}{c^{2}} g_{i k \alpha \beta} \frac{\partial^{2} \Psi}{\partial V_{i k} \partial V_{\alpha \beta}}
$$

This is a canonical equation in a form similar to the Schrodinger equation. It is important what form the Hamiltonian operator can be selected for formulation of the Schrödinger equation for the temporal evolution of the entire Universe. Here the evolution of the wave function passes through the Hypersurface of space-time.

At the time part of the equation takes the integral form.

$$
i h \frac{\partial \Psi}{\partial t}=-\frac{6 \pi G h^{2}}{c^{2}} \int \frac{\partial^{2} \Psi}{\partial V^{2}} d V
$$

It is obvious that the total energy of the universe is definitely in the wording of the question, open or closed universe.

\section{Conclusion}

Perhaps the uncertainty principle between the Hubble parameter and the volume of space has phenomenological applications in cosmology. It is important that this is verifiable result, and future experiments and observations to draw conclusions about the need of the uncertainty principle. In this work we have studied the problem of applying the schrödinger equation to the entire Universe. In fact, the evolution of the wave function of the Universe happens even on the time coordinate.

[1]. Roger Penrose, Foreword: A Computable Universe, Understanding Computation and Exploring Nature As Computation, arxiv: 1205.5823, 2012.

[2] L. Nottale, Fractal spacetime and microphysics, World Scientific, 1993

[3] L. Nottale, Chaos, Solitons, and Fractals, 7 (1996), 877-938; 10 (1999), 459-468; 12 (2000), $1577-$ 1583; 16 (2003), 539-564

[4] H. Ohanian and A. Ruffini, Gravitation and spacetime, Norton, 1994

[5] L. Olavo, Physica A, 262 (1999), 197-214 and 271 (1999), 260-302 and 303-323; Phys. Rev. E, 64 (2001), 036125

[6] R. Parwani, quant-ph 0408185, 0412192, and 0508125 [8] N. Pinto-Neto, gr-qc 0410001, 0410117, and 0410225

[7] N. Pinto-Neto and E. Santini, gr-qc 0009080 and 0302112; Gener. Relativ. Gravitation, 34 (2002), 505; Phys. Lett. A, 315 (2003), 36; Phys. Rev. D, 59 (1999), 123517 (gr-qc 9811067)

[8] S. Popescu, A. Short, and A. Winter, quant-ph 0511225

[9] C. J. Isham, "Topological and global aspects of quantum theory," in Relativ. Groups Topol. II (B. S. DeWitt and R. Stora, eds.). North-Holland, Amsterdam, 1984. 
[10] T. Thiemann, "Complexifier coherent states for quantum general relativity," Class. Quantum Gravity 23 (2006) 2063-2117, arXiv:gr-qc/0206037

[11] S. Hojman, K. Kuchar, and C. Teitelboim, "Geometrodynamics regained," Annals of Physics 96 (1976) 88-135.

[12] N. Bodendorfer, "An embedding of loop quantum cosmology in $(b, v)$ variables into a full theory context," Classical and Quantum Gravity 33 (2016) 125014, arXiv:1512.00713 [gr-qc].

[13] M. Bojowald, U. Büyükçam, S. Brahma, and F. D’Ambrosio, "Hypersurface-deformation algebroids and effective spacetime models," Physical Review D 94 (2016) 104032, arXiv:1610.08355 [gr-qc].

[14] N. Bodendorfer, A. Schäfer, and J. Schliemann, "Holographic signatures of resolved cosmological singularities," arXiv:1612.06679 [hep-th].

[15] N. Bodendorfer and F. Haneder, "Coarse graining as a representation change," Phys. Lett. B 792 (2019) 69-73, arXiv:1811.02792 [gr-qc].

[16] O. Malaeb, "Hamiltonian formulation of mimetic gravity," Physical Review D 91 (2015) 103526, arXiv:1404.4195 [gr-qc].

[17] Josef Kluson, "Canonical Analysis of Inhomogeneous Dark Energy Model and Theory of Limiting Curvature," arXiv:1701.08523 [hep-th].

[18] H. Firouzjahi, M. A. Gorji, and A. H. Mansoori, "Instabilities in Mimetic Matter Perturbations," arXiv:1703.02923 [hep-th].

[19] R. Arnowitt, S. Deser, and C. W. Misner, "Republication of: The dynamics of general relativity," General Relativity and Gravitation 40 (2008) 1997-2027, arXiv:gr-qc/0405109. 